\title{
Atomic-resolution Imaging Using Cs-corrected Vortex Beams
}

\author{
Peter Ercius ${ }^{1}$, Tyler Harvey ${ }^{3}$, Jordan Pierce ${ }^{3}$, Jordan Chess ${ }^{3}$, Martin Linck $^{2}$ and Ben McMorran ${ }^{3}$ \\ 1. National Center for Electron Microscopy, Lawrence Berkeley National Laboratory, Berkeley, CA. \\ 2. Corrected Electron Optical Systems GmbH, Englerstr. 28, D-69126 Heidelberg, Germany. \\ 3. Department of Physics, 1274 University of Oregon, Eugene OR.
}

Phase gratings have been shown to produce electron beams with orbital angular momentum as demonstrated by numerous groups, and show promise for electron magnetic circular dichroism (EMCD) at the atomic scale $[1,2]$. A linear diffraction grating will produce diffracted beams with a separation determined by the pitch or spacing between grating lines. A grating with a central line that forks into $j+1$ central lines will produce a set of diffracted beams each containing discrete units of orbital angular momentum $m=j \times n$ in the $\mathrm{n}^{\text {th }}$ diffraction order [2]. The discontinuity in the center of the grating imparts a "vortex"-type phase on the diffracted beams. We have built such a forked grating with one discontinuity, a radius of $30 \mu \mathrm{m}$, and a grating pitch of $80 \mathrm{~nm}$ using focused ion beam patterning on a 50 $\mathrm{nm}$ thick SiN window. Figure 1 shows a low-magnification SEM image of the grating, and the inset shows the discontinuity (fork) in the center at higher magnification. The SiN thin film was patterned as a phase grating rather than an amplitude grating, and thus the $30 \mathrm{~nm}$ trench depth does not extend through the full thickness of the SiN thin film. Amplitude gratings have a theoretical maximum diffraction efficiency of $10.1 \%$ into the first order, but this grating achieves $\sim 20 \%$ efficiency due to the phase grating design. High diffraction efficiency is a critical consideration for the application of diffractive optics in STEM imaging and spectroscopy.

The phase grating was placed in the second condenser aperture position of the TEAM I Titan 80/300 STEM at the National Center for Electron Microscopy (NCEM). At $300 \mathrm{kV}$, the grating produces a 25.4 mrad probe convergence semi-angle, and $\mathrm{a}^{\text {th }}$ order diffracted beam diverges from the optical axis at an angle of $25 \times \mathrm{n} \mu \mathrm{rad}$. Figure $2 \mathrm{a}$ ) shows a false color image of the $0^{\text {th }}, 1^{\text {st }}$ and $2^{\text {nd }}$ order beams with the post-specimen lenses set to imaging mode for vortex beams with $\mathrm{m}=0, \pm 1, \pm 2$ The $0^{\text {th }}$ order transmitted beam is oversaturated to show the less intense higher order diffracted beams. Each beam is separated by $28 \mathrm{~nm}$ at the sample plane. The vortex phase shift imprinted on the diffracted beams produces a spiral phase singularity with no intensity at the center of a focused beam. This is difficult to image for a sub- $\AA$, $\mathrm{m}=1$ beam even with a post-specimen aberration corrector; however, the convergent beam electron diffraction pattern (CBED) in vacuum of only the beam in figure $2 b$ ) shows the missing intensity in the center as expected. Notice the hard edges in figure $2 b$ ) which is not physically possible for a real space image of the probe.

Isolating one beam for imaging requires a small pre-specimen aperture which is practically difficult considering that the beam must be scanned. High-angle annular dark field (HAADF) images can be acquired if the specimen objects are sufficiently sparse such that only one beam $\left(0^{\text {th }}\right.$ or $1^{\text {st }}$ order $)$ interacts with one object at any probe position. Figure 3 a) shows a single STEM scan showing three representations of the same cluster of FePt nanoparticles. From this image the beam separation, diffraction efficiency and quality of the beams can be analyzed. Figure $3 b$ ) shows an aberrationcorrected image of $\mathrm{SrTiO}_{3}[100]$ with the $0^{\text {th }}$ order (left) and $1^{\text {st }}$ order (right) beams. This demonstrates atomic resolution HAADF-STEM imaging with vortex beams with possible applications to EMCD. 


\section{References:}

[1] J Verbeeck, H Tian and P Schattschneider, Nature, 467 (2010), p. 301 - 304

[2] B McMorran et al., Science 331 (2011), p. 192 - 195

[3] NCEM is supported by the U.S. Department of Energy under contract no. DE-AC02-05CH11231

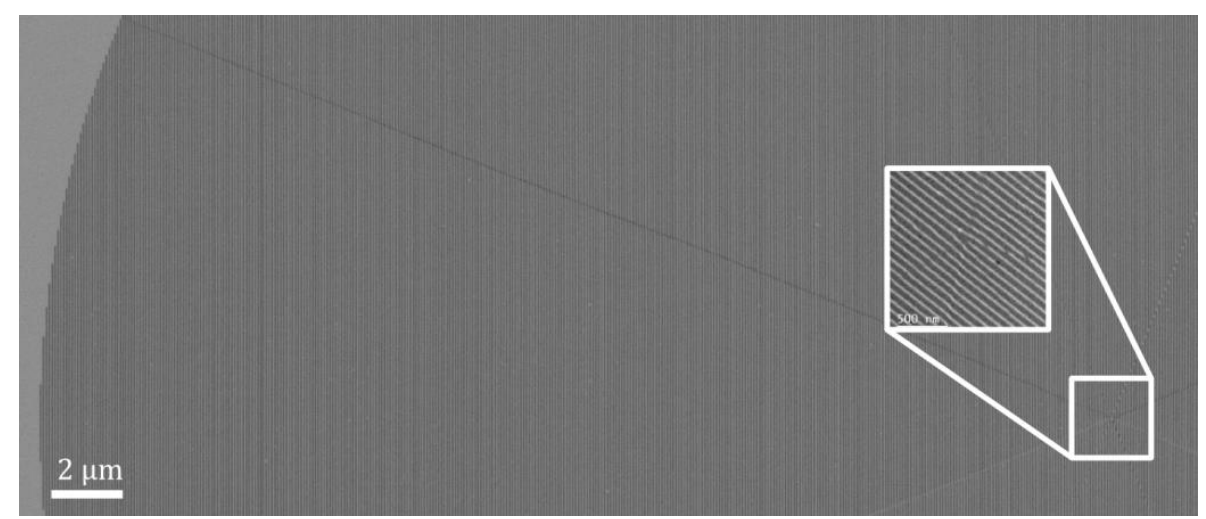

Figure 1. Low-magnification SEM picture of a $30 \square \mathrm{m}$ radius forked grating. Radial lines are for structural integrity. The discontinuity of the fork is shown as an inlay to the figure.

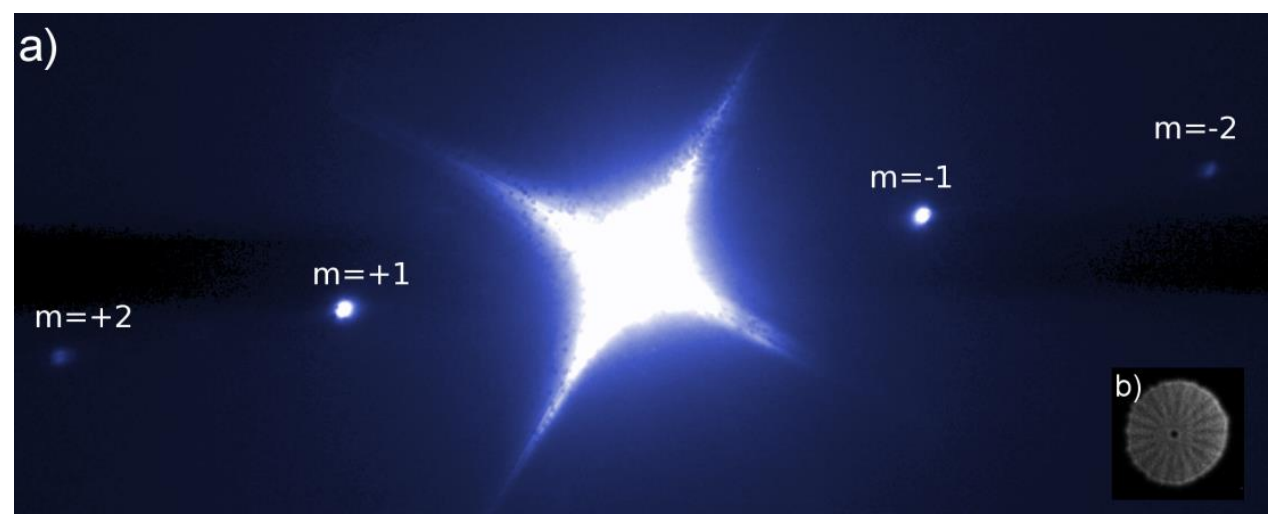

Figure 2. a) False color image of 0 th, 1 st and 2nd diffraction order beams for both the negative and positive orbital angular momentum values. The spacing between the beams is about $28 \mathrm{~nm}$. b) Vacuum CBED of 1st order beam showing a central hole indicating a vortex state.

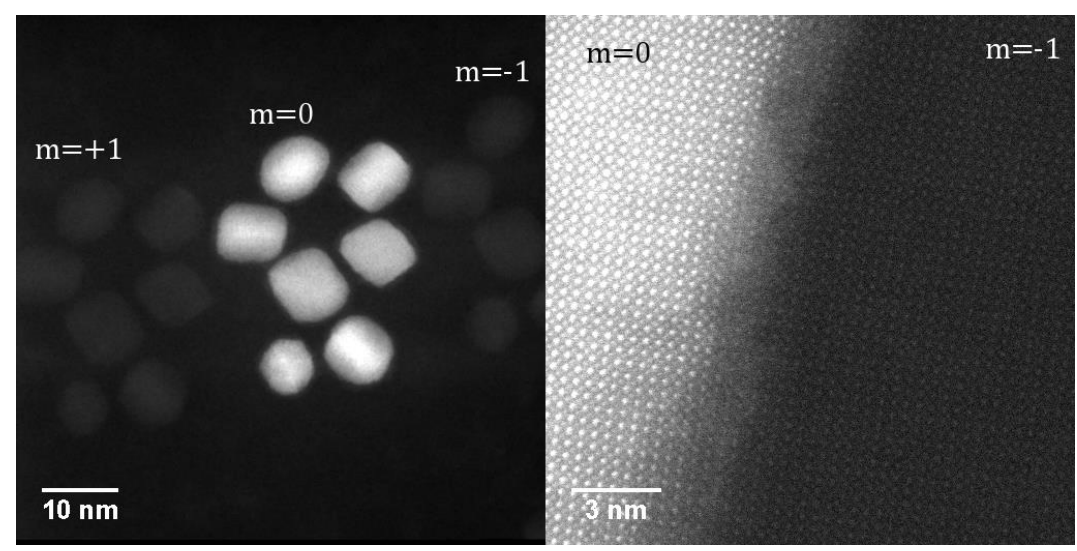

Figure 3. a) HAADF-STEM image of FePt nanoparticles by 0 th and \pm st order beams. b) $\mathrm{SrTiO}_{3}[100]$ imaged at atomic resolution with a 0 th beam (left) and -1 st order beam (right). 